Document downloaded from:

http://hdl.handle.net/10251/169641

This paper must be cited as:

Esteban Romero, R.; Longobardi, P.; Maj, M. (2020). A generalization to Sylow permutability of pronormal subgroups of finite groups. Journal of Algebra and Its Applications. 19(3):1-13. https://doi.org/10.1142/S0219498820500528

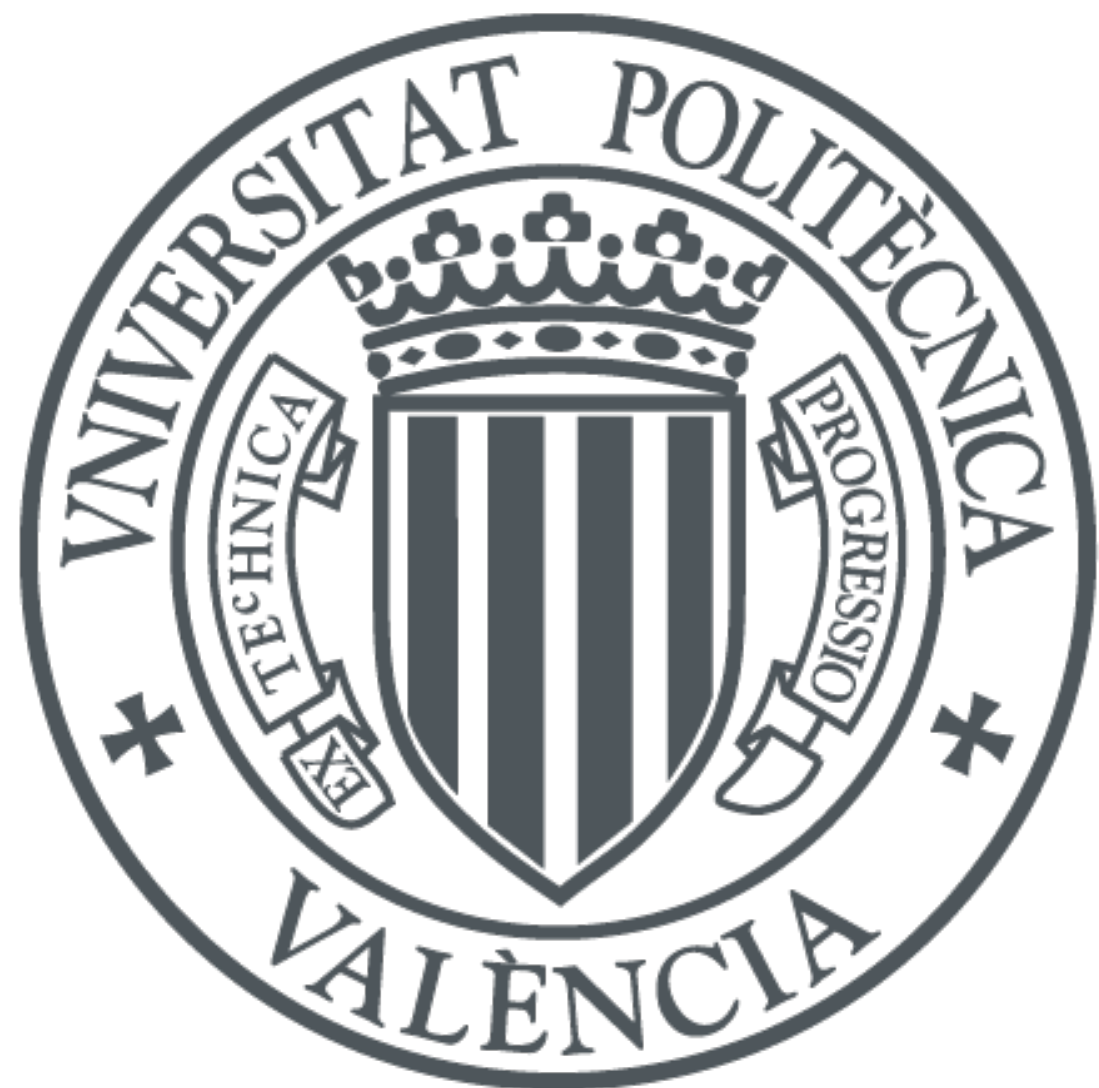

The final publication is available at

https://doi.org/10.1142/S0219498820500528

Copyright World Scientific

Additional Information

Electronic version of an article published as Journal of Algebra and Its Applications, 2020, 19:03 https://doi.org/10.1142/S0219498820500528 (c) World Scientific Publishing Company. 


\title{
A generalisation to Sylow permutability of pronormal subgroups of finite groups
}

\author{
R. Esteban-Romero ${ }^{1} \quad$ P. Longobardi ${ }^{2} \quad$ M. Maj $^{2}$
}

22nd March 2019

Dedicated to the memory of Professor James Clark Beidleman

\begin{abstract}
In this note we present a new subgroup embedding property that can be considered as an analogue of pronormality in the scope of permutability and Sylow permutability in finite groups. We prove that finite PST-groups, or groups in which Sylow permutability is a transitive relation, can be characterised in terms of this property, in a similar way as T-groups, or groups in which normality is transitive, can be characterised in terms of pronormality.

Mathematics Subject Classification (2010): 20D20, 20D35, 20D40, 20D99

Keywords: finite group, subgroup embedding property, permutability, pro-S-permutability, propermutability
\end{abstract}

\section{Introduction}

All groups considered in this paper will be finite.

During the last years, many researchers have analysed groups by studying how all their subgroups or the members of some relevant families of subgroups are embedding into the group. Classes of groups defined in terms of transitivity of a certain subgroup embedding property have attracted a lot

\footnotetext{
${ }^{1}$ Departament de Matemàtiques, Universitat de València, Dr. Moliner, 50, 46100 Burjassot, València, Spain, Ramon.Esteban@uv.es. Permanent address: Institut Universitari de Matemàtica Pura i Aplicada, Universitat Politècnica de València, Camí de Vera, s/n, 46022 València, Spain, resteban@mat.upv.es.

${ }^{2}$ Dipartimento di Matematica, Università degli Studi di Salerno, Via Giovanni Paolo II, 132, 84084 Fisciano, Salerno, Italy, plongobardi@unisa.it, mmaj@unisa.it.
} 
of attention and have been studied in depth for some subgroup embedding properties, like normality, permutability, or Sylow permutability, that give rise to the classes of T-groups, PT-groups, and PST-groups, respectively. In [4, Chapter 2], it has been shown that these three classes are amenable to parallel treatments, especially in the soluble universe. However, the class of soluble T-groups accepts characterisations in terms of pronormality, but no good analogue of these characterisations has been presented so far for PT-groups and PST-groups. The aim of this note is to fill in this gap.

Recall that a subgroup $H$ of a group $G$ is said to be pronormal when given $g \in G$, there exists $x \in\left\langle H, H^{g}\right\rangle$ such that $H^{g}=H^{x}$. This is a well-known subgroup embedding property introduced by Hall in his Cambridge lectures. This property is relevant when we consider families of subgroups of a certain group that form a conjugacy class and that are persistent in intermediate subgroups.

A T-group is a group in which normality is a transitive relation. Many characterisations of T-groups are available, especially in the soluble case. In [2], some characterisations of T-groups in terms of subgroup embedding properties were obtained (see also [4, Chapter 2]). For instance, we have the following characterisation of soluble T-groups.

Theorem 1 ([16, Theorem], [11, Proposition 4]). Let $G$ be a group. The following statements are equivalent:

1. G is a soluble T-group.

2. For every prime number $p$, all p-subgroups of $G$ are pronormal.

3. All subgroups of $G$ are pronormal.

Recall that a subgroup $H$ permutes with a subgroup $K$ when $H K=K H$, that is, $H K$ is a subgroup of $G$. A subgroup $H$ of a group $G$ is said to be permutable in $G$ if $H$ permutes with every subgroup of $G$. Sometimes, this requirement is relaxed so that we are interested in subgroups permuting with a relevant family of subgroups of $G$. This is the case of the S-permutable subgroups: a subgroup $H$ of a group $G$ is said to be $S$-permutable in $G$ if $H$ permutes with all Sylow subgroups of $G$. The classes of PT-groups, or groups in which permutability is a transitive relation, and PST-groups, or groups in which S-permutability is a transitive relation, have been widely studied, especially in the soluble universe. A basic account of these classes of groups can be found in [4, Chapter 2]. Note that the class of PT-groups coincides with the class of groups whose subnormal subgroups are permutable, while the PST-groups are exactly the groups whose subnormal subgroups are Spermutable. 
The aim of this paper is to define two new subgroup embedding properties, that we will call pro-S-permutability and propermutability, and to prove that the analogues of Theorem 1 with T-groups and pronormal subgroups replaced by PST-groups and pro-S-permutable subgroups, respectively, or replaced by PT-groups and propermutable subgroups, respectively, hold. We will also compare these new subgroup embedding properties with other subgroup embedding properties presented in [4, Section 2.3].

\section{$2 \quad$ Subgroup embedding properties characterising T-groups}

In this section, we will recall some subgroup embedding properties that have proved useful to characterise soluble T-, PT-, and PST-groups. We start with the properties characterising soluble T-groups.

Weak normality is an interesting subgroup embedding property introduced by Müller in [14] (see also [4, Definition 1.5.8]).

Definition 2. A subgroup $H$ of a group $G$ is said to be weakly normal whenever for every $g \in G, g \in \mathrm{N}_{G}(H)$ provided that $H^{g} \leqslant \mathrm{~N}_{G}(H)$.

The next property was introduced by Peng in [17]. Mysovskikh gave it the name of subnormaliser condition in [15], while Kurdachenko and Subbotin called the subgroups with this property transitively normal in [13] and de Giovanni and Vincenzi called them pseudonormal in [7] (see also [4, Definition 1.5.13]).

Definition 3. A subgroup $H$ of a group $G$ is said to satisfy the subnormaliser condition if for every subgroup $K$ of $G$ such that $H \unlhd K$, it follows that $\mathrm{N}_{G}(K) \leqslant \mathrm{N}_{G}(H)$.

The following equivalence is clear, as Kurdachenko and Subbotin point out in [13] (see also [4, Proposition 1.5.15]):

Theorem 4. The following statements are equivalent for a subgroup $H$ of a group $G$.

1. H satisfies the subnormaliser condition.

2. If $H$ is a normal subgroup of $K$ and $K$ is a normal subgroup of a subgroup $L$ of $G$, then $H$ is a normal subgroup of $L$.

The following relations between pronormality and the previous concepts hold. 
Theorem 5 ([2], see also [4, Proposition 1.5.9 and 1.5.15]). Let $H$ be $a$ subgroup of a group $G$.

1. If $H$ is pronormal in $G$, then $H$ is weakly normal in $G$.

2. If $H$ is weakly normal in $G$, then $H$ satisfies the subnormaliser condition in $G$.

Moreover, for subgroups of prime power order we have the following equivalences (see [4, Lemma 1.5.17]).

Theorem 6. Let $p$ be a prime and let $H$ be a p-subgroup of $G$. The following statements are equivalent.

1. $H$ is a pronormal subgroup of $G$.

2. $H$ is a weakly normal subgroup of $G$.

3. $H$ satisfies the subnormaliser condition in $G$.

4. $H$ is normal in $\mathrm{N}_{G}(X)$ for every $p$-subgroup $X$ such that $H \leqslant X$.

5. $H$ is normal in $\mathrm{N}_{G}(S)$ for every Sylow p-subgroup $S$ of $G$ such that $H \leqslant S$.

Theorem 7 ([2, Theorem A], see also [4, Theorem 2.3.1]). Let $G$ be a group. Any two of the following conditions are equivalent:

1. $G$ is a soluble T-group.

2. Every subgroup of $G$ is weakly normal in $G$.

3. For every prime number $p$, every p-subgroup of $G$ is weakly normal in $G$.

4. Every subgroup of $G$ satisfies the subnormaliser condition in $G$.

5. For every prime number $p$, every p-subgroup of $G$ satisfies the subnormaliser condition in $G$. 


\section{Subgroup embedding properties characterising PT- and PST-groups}

The parallelism between the classes of T-groups, PT-groups, and PST-groups with respect to the normal structure and the Sylow structure, especially in the soluble universe, was the motivation of [3] (see also [4, Section 2.3]) to obtain characterisations of soluble PT-groups and soluble PST-groups in terms of subgroup embedding properties. The condition " $H^{g} \leqslant \mathrm{~N}_{G}(H)$ " can be interpreted as " $H$ is a normal subgroup of $\left\langle H, H^{g}\right\rangle$ ", while the condition " $g \in \mathrm{N}_{G}(H)$ " can be interpreted as " $H$ is a normal subgroup of $\langle H, g\rangle$ ". This motivated the introduction of the following concepts in [3] (see also [4, Section 2.3]) by replacing the words "normal" by "permutable" and "Spermutable", respectively.

Definition 8. A subgroup $H$ of a group $G$ is said to be weakly permutable (respectively, weakly $S$-permutable) in $G$ when the following condition holds: if $g \in G$ and $H$ is permutable (respectively, S-permutable) in $\left\langle H, H^{g}\right\rangle$, then $H$ is permutable (respectively, S-permutable) in $\langle H, g\rangle$.

Definition 9. A subgroup $H$ of a group $G$ satisfies the subpermutiser condition (respectively, satisfies the S-subpermutiser condition) if the following condition holds: if $H$ is permutable (respectively, S-permutable) in $K$ and $x$ is an element of $G$ such that $K$ is permutable (respectively, S-permutable) in $\langle K, x\rangle$, then $H$ is permutable (respectively, $\mathrm{S}$-permutable) in $\langle H, x\rangle$.

The following relations between the above concepts are satisfied ([3], see also [4, Section 2.3]).

Proposition 10. 1. Every weakly normal subgroup is weakly permutable.

2. Every weakly permutable subgroup is weakly S-permutable.

3. Every subgroup satisfying the subnormaliser condition satisfies the subpermutiser condition.

4. Every subgroup satisfying the subpermutiser condition satisfies the $S$ subpermutiser condition

5. Every weakly permutable subgroup satisfies the subpermutiser condition.

6. Every weakly S-permutable subgroup satisfies the S-subpermutiser condition. 
Indeed, the fact that $H$ satisfies the subpermutiser condition (respectively, the S-subpermutiser condition) in $G$ is equivalent to affirming that if $H$ is subnormal in a subgroup $K$ of $G$, then $H$ is permutable (respectively, Spermutable) in $K$, as pointed out in [4, Corollary 2.3.5].

\section{Extensions of pronormality to permutability and Sylow permutability}

In this section, we will obtain an analogue of pronormality to permutability and S-permutability in such a way we can find an analogue of Theorems 1 and 5. It will be based on the following characterisation of pronormality, which was shown in [6], in a remark after Definition 2. For completeness, we give a proof of this result.

Lemma 11. Let $H$ be a subgroup of $G$. Then $H$ is pronormal in $G$ if and only if for each $g \in G$ there exists $x \in\left\langle H, H^{g}\right\rangle^{\mathfrak{N}}$ such that $H^{g}=H^{x}$.

Here $X^{\mathfrak{N}}$ denotes the nilpotent residual of a group $X$, that is, the smallest normal subgroup $N$ of $X$ such that $X / N$ is nilpotent. This subgroup coincides with the limit of the lower central series of $X$.

Proof of Lemma 11. Suppose that $H$ is pronormal in $G$, let $g \in G$, and call $T=\left\langle H, H^{g}\right\rangle$. Now the group $T / T^{\mathfrak{N}}$ is nilpotent. Since pronormality behaves well with respect to intermediate subgroups and epimorphic images, $H T^{\mathfrak{N}} / T^{\mathfrak{N}}$ is pronormal in the nilpotent group $T / T^{\mathfrak{N}}$. Moreover, all subgroups of $T / T^{\mathfrak{N}}$ are subnormal. Since a subgroup that is pronormal and subnormal is normal, we obtain that $H T^{\mathfrak{N}}$ is normal in $T$. There exists $x \in T$ such that $H^{g}=H^{x}$. Therefore $H T^{\mathfrak{N}}=H^{x} T^{\mathfrak{N}}=H^{g} T^{\mathfrak{N}}$ and so $T=\left\langle H, H^{g}\right\rangle \leq H T^{\mathfrak{N}}$. Consequently, $T=H T^{\mathfrak{N}}$. It follows that there exist $h \in H$ and $y \in T^{\mathfrak{N}}$ such that $x=h y$, hence $H^{x}=H^{h y}=H^{y}$ with $y \in T^{\mathfrak{N}}$.

In order to find this analogue, we rewrite the condition for the pronormality of $H$ in $G$ given in Lemma 11 in the form "for every $g \in G$ there exists $x \in\left\langle H, H^{g}\right\rangle^{\mathfrak{N}}$ such that $H^{g x^{-1}}=H$ ", that is, "for every $g \in G$ there exists $x \in\left\langle H, H^{g}\right\rangle^{\mathfrak{N}}$ such that $H$ is normal in $\left\langle H, g x^{-1}\right\rangle^{\prime \prime}$. This motivates the following definitions.

Definition 12. A subgroup $H$ of a group $G$ is said to be propermutable ${ }^{1}$ in $G$ when for every $g \in G$ there exists $x \in\left\langle H, H^{g}\right\rangle^{\mathfrak{N}}$ such that $H$ is permutable in $\left\langle H, g x^{-1}\right\rangle$.

\footnotetext{
${ }^{1}$ We warn that the word propermutable has also been used by Yi and Skiba with a different meaning in $[20]$.
} 
Definition 13. A subgroup $H$ of a group $G$ is said to be pro-S-permutable in $G$ when for every $g \in G$ there exists $x \in\left\langle H, H^{g}\right\rangle^{\mathfrak{N}}$ such that $H$ is Spermutable in $\left\langle H, g x^{-1}\right\rangle$.

The following result is immediate from these definitions.

Proposition 14. 1. Every pronormal subgroup is propermutable.

2. Every propermutable subgroup is pro-S-permutable.

Pro-S-permutability and propermutability satisfy properties analogous to the one stated in Theorem 5 .

Theorem 15. Every pro-S-permutable (respectively, propermutable) subgroup is weakly S-permutable (respectively weakly permutable).

Proof. We will prove the result for pro-S-permutable subgroups, the proof for propermutable subgroups is similar. Assume that $H$ is pro-S-permutable in $G$. Let $g \in G$ and assume that $H$ is S-permutable in $T=\left\langle H, H^{g}\right\rangle$. Since $H$ is pro-S-permutable in $G$, there exists $x \in T^{\mathfrak{N}}$ such that $H$ is S-permutable in $\left\langle H, g x^{-1}\right\rangle$. Now $T^{\mathfrak{N}}=\bigcap_{p \in \mathbb{P}} \mathrm{O}^{p}(T)$. Since $H$ is S-permutable in $T$, we obtain that $H / H_{T}$ is S-permutable in $T / H_{T}$. By a result of Deskins [8] and Kegel [12] (see [4, Theorem 1.2.14 (2)]), $H / H_{T}$ is a subnormal nilpotent subgroup of $T / H_{T}$. Let $H / H_{T}=\prod_{p \in \mathbb{P}}\left(H_{p} / H_{T}\right)$, where $H_{p} / H_{T}$ is a Sylow $p$-subgroup of $H / H_{T}$. By a result of Schmid [18] (see [4, Theorem 1.2.17]), $H_{p} / H_{T}$ is Spermutable in $T / H_{T}$. By [4, Lemma 1.2.16], we conclude that $\mathrm{O}^{p}\left(T / H_{T}\right) \leq$ $\mathrm{N}_{T / H_{T}}\left(H_{p} / H_{T}\right)$ for each prime $p$. But since $\mathrm{O}^{p}\left(T / H_{T}\right)=\mathrm{O}^{p}(T) H_{T} / H_{T}$ and $x \in \mathrm{O}^{p}(T)$ for each prime $p$, we have that $H_{p}^{x} / H_{T}=H_{p} / H_{T}$. In particular, $H^{x}=H$. Now $H^{g^{-1}}=H^{x g^{-1}} \leq\left\langle H, g x^{-1}\right\rangle$. Moreover, $g x g^{-1}=x^{g^{-1}} \in$ $\left\langle H, H^{g^{-1}}\right\rangle$ and so

$$
\begin{aligned}
\left\langle H, g x^{-1}\right\rangle & =\left\langle H, H^{g^{-1}}, g x^{-1}\right\rangle=\left\langle H, H^{g^{-1}}, g x g^{-1}, g x^{-1}\right\rangle \\
& =\left\langle H, H^{g^{-1}}, g, g x^{-1}\right\rangle=\langle H, g\rangle .
\end{aligned}
$$

This completes the proof of the theorem.

Proposition 14 and Theorem 15 can be used to complete Figure 1. The following three examples show that the new implications in this diagram are strict.

Example 16. Let $G$ be an Iwasawa $p$-group that is not Dedekind. A nonnormal subgroup $H$ of $G$ is propermutable, since it is permutable, but is not pronormal, because it is subnormal and so it would be normal. 


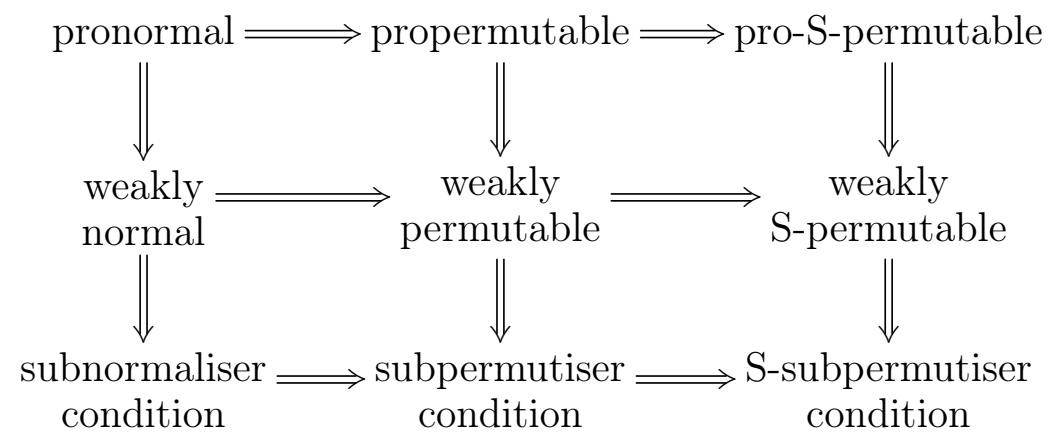

Figure 1: Subgroup embedding properties and their relations

Example 17. The non-normal subgroups of the dihedral group of order 8 are pro-S-permutable, since they are S-permutable, but are not propermutable, because as they are subnormal they would be permutable.

Example 18. Let $V$ be an irreducible and faithful module for the symmetric $\Sigma_{3}$ of degree 3 over the field $\mathrm{GF}(7)$ of 7 elements such that the restriction fo $V$ to the alternating group $A_{3}$ is a direct sum of two irreducible and faithful $A_{3}$-submodules $W_{1}$ and $W_{2}$ of dimension 1 . Let $G=[V] \Sigma_{3}$ be the corresponding semidirect product and let $H=\left[W_{1}\right] A_{3}$. As in [2, Remark 1] (see also [4, Example 1.5.10]), the subgroup $H$ is weakly normal in $G$. Let $a$ be an element of order 2 in $\Sigma_{3}$. We can check that if $H$ is permutable in $\langle H, x\rangle$ with $x \in G$, then $x \in H$. In particular, $H$ cannot be pro-S-permutable in $G$.

There are examples of groups with a $p$-subgroup which is not weakly normal but with all $p$-subgroups satisfying the subnormaliser condition. One of them appears in [3, Example 2] (see also [4, Example 2.3.9]). Therefore, the equivalence between the conditions 2 and 3 in Theorem 6 cannot be extended to permutability or S-permutability. However, we have the following result.

Theorem 19. Let $p$ be a prime and let $H$ be a p-subgroup of $G$. If $H$ is weakly permutable (respectively, weakly $S$-permutable) in $G$, then $H$ is propermutable (respectively, pro-S-permutable) in $G$.

Our proof of Theorem 19 depends on the fact that pro-S-permutable (respectively, propermutable) subgroups satisfy the S-subpermutiser (respectively, the subpermutiser) condition. We prove first a new characterisation of subgroups satisfying the S-subpermutiser condition.

Theorem 20. The following statements are equivalent about a subgroup $H$ of a group $G$ : 


\section{1. $H$ satisfies the $S$-subpermutiser condition in $G$.}

2. If $H$ is S-permutable in $\left\langle H^{\langle H, g\rangle}\right\rangle$, then $H$ is $S$-permutable in $\langle H, g\rangle$.

Proof. Assume that 1 holds and that $H$ is S-permutable in $T=\left\langle H^{\langle H, g\rangle}\right\rangle$. Since $T \unlhd\langle H, g\rangle$, we obtain that $H$ is subnormal in $\langle H, g\rangle$. By the Ssubpermutisiser condition, $H$ is S-permutable in $\langle H, g\rangle$.

Now assume that 2 holds. By induction, we can suppose that $H$ is $\mathrm{S}$ permutable in $K$ and that $K$ is normal in $L$. Let $g \in L$. Then $\left\langle H^{\langle H, g\rangle}\right\rangle \leqslant K$ and so $H$ is S-permutable in $\left\langle H^{\langle H, g\rangle}\right\rangle$. Hence $H$ is S-permutable in $\langle H, g\rangle$. Since this holds for all $g \in G$, we conclude by [4, Theorem 1.2.13] that $H$ is S-permutable in $L$.

Theorem 21. Assume that $H$ is pro-S-permutable in $G$. Then $H$ satisfies the $S$-subpermutiser condition in $G$.

Proof. We will use Theorem 20. Assume that the result is false. Let $G$ be a group with a pro-S-permutable subgroup $H$ such that $H$ is S-permutable in $\left\langle H^{\langle H, g\rangle}\right\rangle$, but $H$ is not S-permutable in $\langle H, g\rangle$, in which $|G|$ and $|\langle H, g\rangle|$ are as small as possible.

Let $Y$ be a proper subgroup of $G$ contained in $\langle H, g\rangle$. We have that $H$ is pro-S-permutable in $Y$. Let $y \in Y$. Since $|Y|<|G|, Y$ satisfies the thesis of the theorem. Hence, if $y \in Y$ and $H$ is S-permutable in $\left\langle H^{\langle H, y\rangle}\right\rangle$, then $H$ is S-permutable in $\langle H, y\rangle$. But $\langle H, y\rangle \leqslant Y \leqslant\langle H, g\rangle$. Since $H$ is S-permutable in $\left\langle H^{\langle H, g\rangle}\right\rangle$, it turns out that $H$ is S-permutable in its subgroup $\left\langle H^{\langle H, y\rangle}\right\rangle$. Therefore $H$ is S-semipermutable in $\langle H, y\rangle$. By [4, Theorem 1.2.13], $H$ is S-permutable in $Y$. In particular, if $\langle H, g\rangle\langle G$, then we have that $H$ is S-permutable in $\langle H, g\rangle$, against the hypothesis. Hence $\langle H, g\rangle=G$. Since $H$ is S-permutable in $\left\langle H^{\langle H, g\rangle}\right\rangle=\left\langle H^{G}\right\rangle, H$ is subnormal in $\left\langle H^{G}\right\rangle$ by [12] (see also [4, Theorem 1.2.14]). Since $\left\langle H^{G}\right\rangle$ is a normal subgroup of $G$, we conclude that $H$ is subnormal in $G$.

Let $M$ be a maximal normal subgroup of $G$ containing $H$. Of course, since $H$ is not S-permutable in $G, H \neq 1$ and so $M \neq 1$. Let $N$ be a minimal normal subgroup of $G$ contained in $M$. Since $H N / N$ is pro-S-permutable in $G / N$ and $H N / N$ is S-permutable in $\left\langle(H N / N)^{\langle H N / N, g N\rangle}\right\rangle=\left\langle(H N / N)^{G / N}\right\rangle$, by the minimality of $G$ it turns out that $H N / N$ is S-permutable in $\langle H N / N, g N\rangle=$ $G / N$. In particular, $H N$ is S-permutable in $G$. But now $H$ is not Spemutable in $G$. This means that there exists a prime $p$ and a Sylow $p$ subgroup $P$ of $G$ such that $H$ does not permute with $P$. Since $H N / N$ is S-permutable in $\langle H N / N, g N\rangle$, we conclude that $H N / N$ permutes with $P N / N$, that is, $(H N) P$ is a subgroup of $G$. If $(H N) P$ is a proper subgroup of $G$, we obtain that $T=(H N) P$ satisfies the hypothesis of the theorem by 
minimality of $G$ and so $H$ is S-permutable in $T$, in particular, $H$ permutes with $P$. Therefore $(H N) P=G$. Now let $M_{1}$ be a maximal subgroup of $G$ containing $H$ such that $M$ is not contained in $M_{1}$. Then $M M_{1}=G$. By [10, Kapitel IV, Satz 4.6], there exist Sylow $p$-subgroups $Q$ of $M$ and $Q_{1}$ of $M_{1}$ such that $P_{0}=Q Q_{1}$ is a Sylow $p$-subgroup of $G$. Hence there exists an element $z \in G$ such that $P_{0}=P^{z}$. Since $G=(H N) P=P(H N)$, there exist $z_{1} \in P$ and $z_{2} \in H N$ such that $z=z_{1} z_{2}$. Consequently $P_{0}=P^{z_{2}}$. But $H$ permutes with $P^{z_{2}}$, that is, $H^{z_{2}^{-1}}$ permutes with $P$. By a result of Wielandt [19] (see also [9, Chapter 3, Lemma 14.3]), $N$ normalises $H$ and so $H^{z_{2}^{-1}}=H$ permutes with $P$. This contradiction shows that every maximal subgroup of $G$ containing $H$ must contain $M$. Since $G=(H N) P=M P,|G: M|$ is a power of $p$. It follows that all maximal subgroups of $G / M$ are normal, in particular, $M$ is itself a maximal subgroup of $G$. Now there exists an element $x \in\left\langle H, H^{g}\right\rangle$ such that $H$ is S-permutable in $\left\langle H, g x^{-1}\right\rangle$. The normality of $M$ implies that $\left\langle H, H^{g}\right\rangle \leqslant M$ and so $x \in M$. Assume now that $\left\langle H, g x^{-1}\right\rangle$ is a proper subgroup of $G$. Then $\left\langle H, g x^{-1}\right\rangle$ is contained in a maximal subgroup of $G$, that must be exactly $M$. It follows that $g x^{-1} \in M$. But then $g \in M$, which implies that $G=\langle H, g\rangle \leqslant M$. This contradiction completes the proof of the theorem.

Now we are in a position to give a proof of Theorem 19.

Proof of Theorem 19. We will give the proof for S-permutability, the proof for permutability is analogous. Let $H$ be a weakly S-permutable $p$-subgroup of $G$ and let $g \in G$. Let $T=\left\langle H, H^{g}\right\rangle$. Let $P_{0}$ be a Sylow $p$-subgroup of $T$. The unique Sylow $p$-subgroup of the nilpotent group $T / T^{\mathfrak{N}}$ is then $P_{0} T^{\mathfrak{N}} / T^{\mathfrak{N}}$ and, since $H$ and $H^{g}$ are $p$-subgroups of $T, H T^{\mathfrak{N}} / T^{\mathfrak{N}}$ and $H^{g} T^{\mathfrak{N}} / T^{\mathfrak{N}}$ are contained in $P_{0} T^{\mathfrak{N}} / T^{\mathfrak{N}}$. Consequently $T=\left\langle H, H^{g}\right\rangle \leq P_{0} T^{\mathfrak{N}}$, which implies that $T=P_{0} T^{\mathfrak{N}}$. Let $P$ be a Sylow $p$-subgroup of $G$ such that $P_{0} \leq P$. Then $H^{g}$ is contained in a conjugate of $P_{0}$ in $T$, that is, there exist $z \in P_{0}$, $x \in T^{\mathfrak{N}}$ such that $H^{g} \leq P_{0}^{z x}=P_{0}^{x}$. In particular, $H^{g x^{-1}} \leq P_{0} \leq \mathrm{N}_{G}(P)$. Hence $H \leq\left\langle H, H^{g x^{-1}}\right\rangle \leq \mathrm{N}_{G}(P)$. Since $H$ satisfies the S-subpermutiser condition by Theorem 20 and $H$ is subnormal in $\mathrm{N}_{G}(P)$, we have that $H$ is Spermutable in $\mathrm{N}_{G}(P)$. In particular, $H$ is S-permutable in $\left\langle H, H^{g x^{-1}}\right\rangle$. Since $H$ is weakly S-permutable, we obtain that $H$ is S-permutable in $\left\langle H, g x^{-1}\right\rangle$. Hence $H$ is pro-S-permutable in $G$.

In [3, Theorem C] (see also [4, Theorem 2.3.8]), the following characterisation of soluble PST-groups was presented.

Theorem 22. Let $G$ be a group. The following statements are equivalent:

1. G is a soluble PST-group. 
2. Every subgroup of $G$ is weakly $S$-permutable in $G$.

3. For every prime number $p$, every p-subgroup of $G$ is weakly $S$-permutable in $G$.

4. Every subgroup of $G$ satisfies the $S$-subpermutiser condition in $G$.

5. For every prime number $p$, every $p$-subgroup of $G$ satisfies the $S$-subpermutiser condition in $G$.

We can extend Theorem 22 to include also pro-S-permutability.

Theorem 23. Let $G$ be a group. The following statements are equivalent:

1. $G$ is a soluble PST-group.

2. Every subgroup of $G$ is pro-S-permutable.

3. For every prime number $p$, every $p$-subgroup of $G$ is pro-S-permutable.

In the proof of Theorem 23 we will use the following elementary result.

Lemma 24. Suppose that $N$ is a normal subgroup of $G$ contained in $H$ and that $H / N$ is pro-S-permutable in $G / N$. Then $H$ is pro-S-permutable in $G$.

Proof. Let $g \in G$. By hypothesis, there exists $x N \in\left\langle H / N,(H / N)^{g N}\right\rangle^{\mathfrak{N}}$ such that $H / N$ is S-permutable in $\left\langle H / N, g x^{-1} N\right\rangle$. Since $\left\langle H / N,(H / N)^{g N}\right\rangle^{\mathfrak{N}}=$ $\left\langle H / N, H^{g} / N\right\rangle^{\mathfrak{N}}=\left\langle H, H^{g}\right\rangle^{\mathfrak{N}} N / N$, we can assume without loss of generality that $x \in\left\langle H, H^{g}\right\rangle^{\mathfrak{N}}$. Moreover, $\left\langle H / N, g x^{-1} N\right\rangle=\left\langle H, g x^{-1}\right\rangle / N$. By [4, Theorem 1.2.14 (1)], the result holds.

Proof of Theorem 23. It is obvious that 2 implies 3. By Theorem 19, if for every prime number $p$, every $p$-subgroup of $G$ is pro-S-permutable, then, for every prime number $p$, every $p$-subgroup of $G$ is weakly S-permutable. By Theorem 22, $G$ is a soluble PST-group.

Let us prove now that 1 implies 2. Suppose that $G$ is a soluble PSTgroup. We will prove the result by induction on the order of $G$. Let $H$ be a subgroup of $G$. If $H_{G} \neq 1$, we can use the induction hypothesis on $G / H_{G}$ to conclude that $H / H_{G}$ is pro-S-permutable in $G / H_{G}$ and so $H$ is pro-Spermutable in $G$ by Lemma 24. Hence we can assume that $H_{G}=1$. By Agrawal's theorem, the nilpotent residual $L$ of $G$ is an abelian Hall subgroup of odd order of $G$ such that $G$ acts on $L$ as a group of power automorphisms. By [5, Theorem 4.2.17], $L$ is complemented in $G$ by a system normaliser $D$, which is a nilpotent Hall subgroup of $G$, and all such complements are conjugate. Since $H \cap L$ is normal in $G$, we have that $H \cap L=1$. Let $\pi$ be 
the set of primes dividing $|L|$. Then $H$ is a $\pi^{\prime}$-subgroup. Let $T=\left\langle H, H^{g}\right\rangle$. Let $D_{0}$ be a Hall $\pi^{\prime}$-subgroup of $T$ containing $H$. Since $T / T^{\mathfrak{N}}$ is nilpotent, $D_{0} T^{\mathfrak{N}} / T^{\mathfrak{N}}$ is the unique Hall $\pi^{\prime}$-subgroup of $T / T^{\mathfrak{N}}$, and it must contain both $H T^{\mathfrak{N}} / T^{\mathfrak{N}}$ and $H^{g} T^{\mathfrak{N}} / T^{\mathfrak{N}}$. Consequently $T / T^{\mathfrak{N}}=\left\langle H, H^{g}\right\rangle / T^{\mathfrak{N}} \leq D_{0} T^{\mathfrak{N}} / T^{\mathfrak{N}}$ and so $T=D_{0} T^{\mathfrak{N}}$. By the dominance of the Hall $\pi^{\prime}$-subgroups in soluble groups, there exist $d \in D_{0}$ and $x \in T^{\mathfrak{N}}$ such that $H^{g} \leq D_{0}^{d x}=D_{0}^{x}$. Therefore $H^{g x^{-1}} \leq D_{0}$. Since $D_{0}$ is nilpotent, all its subgroups are S-permutable, so $H$ is S-permutable in $D_{0}$. Since $H \leq\left\langle H, H^{g x^{-1}}\right\rangle \leq D_{0}, H$ is S-permutable in $\left\langle H, H^{g x^{-1}}\right\rangle$. Since $G$ is a PST-group, $H$ is weakly S-permutable in $G$ by Theorem 22. It follows that $H$ is S-permutable in $\left\langle H, g x^{-1}\right\rangle$. This proves that $H$ is pro-S-permutable in $G$.

The same proof shows that the following characterisation of soluble PTgroups also holds.

Theorem 25. Let $G$ be a group. The following statements are equivalent:

1. G is a soluble PT-group.

2. Every subgroup of $G$ is propermutable.

3. For every prime number $p$, every p-subgroup of $G$ is propermutable.

The following result improves [3, Theorem B] (see also [4, Theorem 2.3.7]), where it was shown that the equivalence holds if every subgroup satisfies one of both conditions.

Theorem 26. Let $p$ be a prime. For a p-subgroup $H$ of $G$, the following statements are equivalent.

1. If $H$ is contained in a p-subgroup $K$ of $G$, then $H$ is $S$-permutable (respectively, permutable) in $\mathrm{N}_{G}(K)$.

2. $H$ satisfies the $S$-subpermutiser condition (respectively, the subpermutiser condition) in $G$.

Proof. We will prove the result for S-permutability, since the proof for permutability is analogous.

Assume that 1 holds. Suppose that $H$ is a subnormal subgroup of a subgroup $L$ of $G$. Then $H$ is contained in $\mathrm{O}_{p}(L)$ by hypothesis. Since $\mathrm{O}_{p}(L)$ is a $p$-subgroup, $H$ is $\mathrm{S}$-permutable in $\mathrm{N}_{G}\left(\mathrm{O}_{p}(L)\right)$. Since $L \leq \mathrm{N}_{G}\left(\mathrm{O}_{p}(L)\right)$, we conclude that $H$ is S-permutable in $L$. Hence 2 holds.

Assume now that 2 holds. Suppose that $H$ is contained in a $p$-subgroup $K$ of $G$. Then $H$ is subnormal in $K$ and so $H$ is subnormal in $\mathrm{N}_{G}(K)$. By hypothesis, $H$ is S-permutable in $\mathrm{N}_{G}(K)$. This proves that 1 holds. 


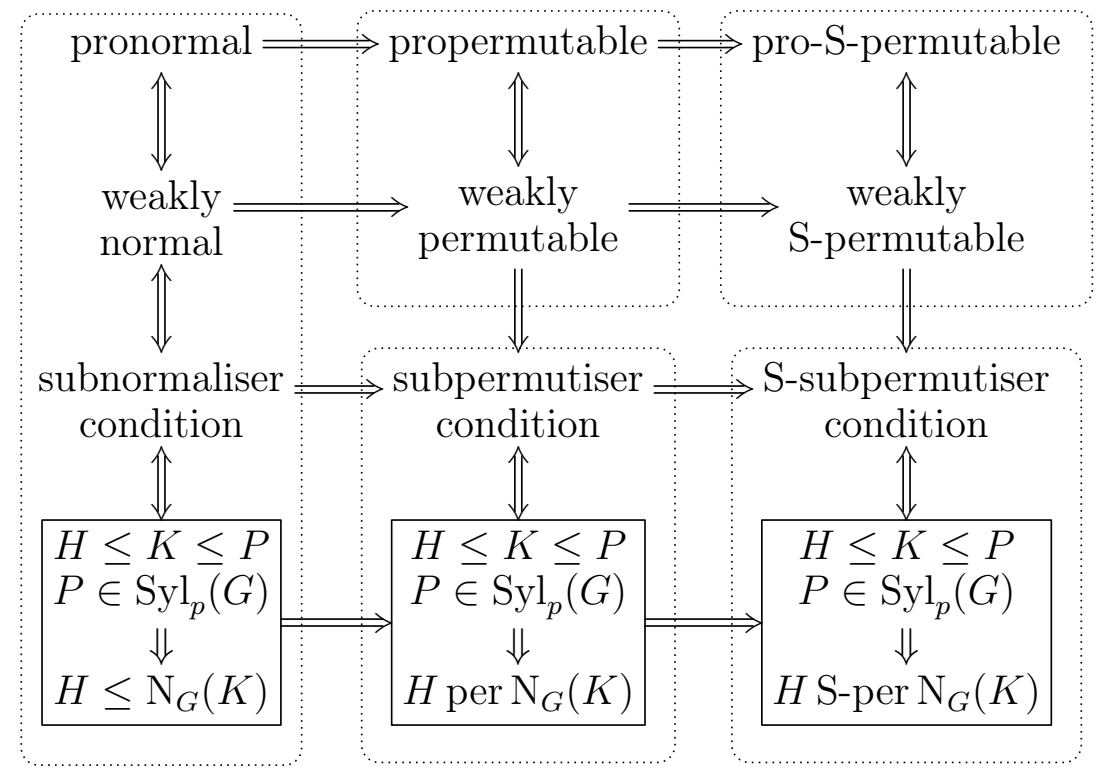

Figure 2: Subgroup embedding properties and their relations for a given $p$-subgroup $H$ of $G$

The relations between these subgroup embedding properties for $p$-subgroups can be summarised in a graphical form in Figure 2.

\section{Acknowledgements}

This work was supported by the "National Group for Algebraic and Geometric Structures, and their Applications" (GNSAGA - INdAM), Italy.

The research of the first author has been supported by the research grants MTM2014-54707-C3-1-P by the "Ministerio de Economía y Competitividad" (Spain) and FEDER (European Union) and PROMETEO/2017/057 from "Generalitat" (Valencian Community, Spain). Part of the work of this paper has been done during some visits of the first author to the Dipartimento di Matematica of the Università degli Studi di Salerno supported by the "National Group for Algebraic and Geometric Structures, and their Applications" (GNSAGA - INdAM), Italy.

\section{References}

[1] A. Ballester-Bolinches and R. Esteban-Romero. Sylow permutable subnormal subgroups of finite groups. J. Algebra, 251(2):727-738, 2002. 
[2] A. Ballester-Bolinches and R. Esteban-Romero. On finite $\mathcal{T}$-groups. $J$. Austral. Math. Soc. Ser. A, 75:1-11, 2003.

[3] A. Ballester-Bolinches and R. Esteban-Romero. On finite soluble groups in which Sylow permutability is a transitive relation. Acta Math. Hungar., 101(3):193-202, 2003.

[4] A. Ballester-Bolinches, R. Esteban-Romero, and M. Asaad. Products of finite groups, volume 53 of de Gruyter Expositions in Mathematics. Walter de Gruyter GmbH \& Co. KG, Berlin, 2010.

[5] A. Ballester-Bolinches and L. M. Ezquerro. Classes of Finite Groups, volume 584 of Mathematics and Its Applications. Springer, Dordrecht, 2006 .

[6] A. Ballester-Bolinches, A. D. Feldman, M. C. Pedraza-Aguilera, and M. F. Ragland. A class of generalised finite T-groups. J. Algebra, 333(1):128-138, 2011.

[7] F. de Giovanni and G. Vincenzi. Pseudonormal subgroups of groups. Ricerche Mat., 52(1):91-101, 2003.

[8] W. E. Deskins. On quasinormal subgroups of finite groups. Math. Z., $82: 125-132,1963$.

[9] K. Doerk and T. Hawkes. Finite soluble groups, volume 4 of De Gruyter Expositions in Mathematics. Walter de Gruyter \& Co., Berlin, 1992.

[10] B. Huppert. Endliche Gruppen I, volume 134 of Grund. Math. Wiss. Springer Verlag, Berlin, Heidelberg, New York, 1967.

[11] G. Kaplan. On T-groups, supersolvable groups, and maximal subgroups. Arch. Math. (Basel), 96(1):19-25, 2011.

[12] O. H. Kegel. Sylow-Gruppen und Subnormalteiler endlicher Gruppen. Math. Z., 78:205-221, 1962.

[13] L. A. Kurdachenko and I. Y. Subbotin. Transitivity of normality and pronormal subgroups. In B. Fine et al., editors, Combinatorial group theory, discrete groups, and number theory. A conference in honor of Gerhard Rosenberger's sixtieth birthday, Fairfield, CT, USA, December 8-9, 2004 and the AMS special session on infinite groups, Bard College, Annandale-on-Hudson, NY, USA, October 8-9, 2005, volume 421 of Contemporary Mathematics, pages 201-212, Providence, RI, 2006. American Mathematical Society (AMS). 
[14] K. H. Müller. Schwachnormale Untergruppen: Eine gemeinsame Verallgemeinerung der normalen und normalisatorgleichen Untergruppen. Rend. Sem. Mat. Univ. Padova, 36:129-157, 1966.

[15] V. I. Mysovskikh. Investigation of subgroup embeddings by the computer algebra package GAP. In Computer algebra in scientific computing-CASC'99 (Munich), pages 309-315, Berlin, 1999. Springer.

[16] T. A. Peng. Finite groups with pronormal subgroups. Proc. Amer. Math. Soc., 20:232-234, 1969.

[17] T. A. Peng. Pronormality in finite groups. J. London Math. Soc. (2), 3:301-306, 1971.

[18] P. Schmid. Subgroups permutable with all Sylow subgroups. J. Algebra, 207:285-293, 1998.

[19] H. Wielandt. Eine Verallgemeinerung der invarianten Untergruppen. Math. Z., 45:209-244, 1939.

[20] X. Yi and A. N. Skiba. On s-propermutable subgroups of finite groups. Bull. Malays. Math. Sci. Soc., 38(2):605-616, 2015. 